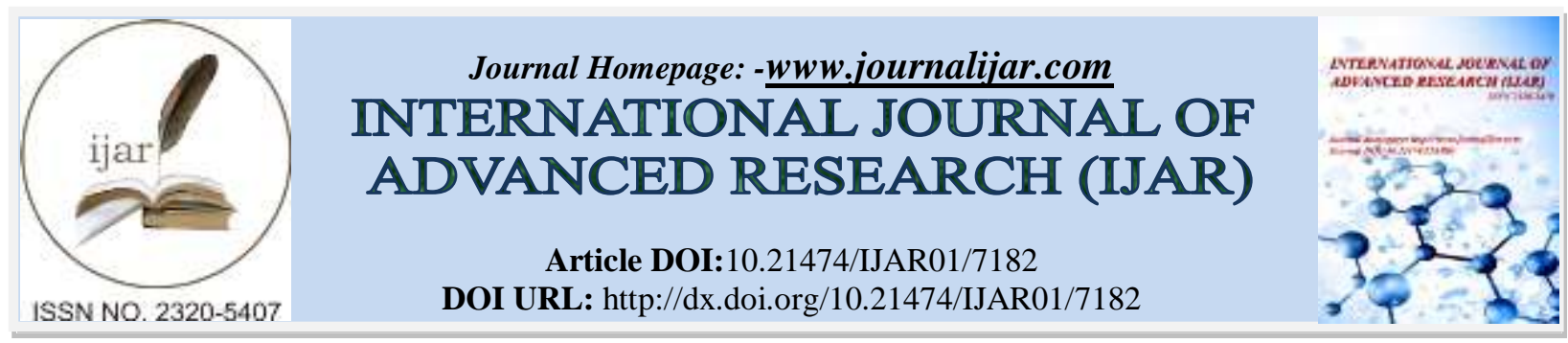

RESEARCH ARTICLE

\title{
PRIMARY LYMPHEDEMA AND PSORIASIS: ETIOPATHOGENIC SIMILARITY AND THERAPEUTIC HOPES.
}

Jawad El-azhari, Naoufal Hjira and Mohammed Boui.
Service de dermatologie-vénérologie, Hôpital d'instruction Mohammed V, Rabat-Maroc.

\section{Manuscript Info}

Manuscript History

Received: 02 April 2018

Final Accepted: 04 May 2018

Published: June 2018

Keywords:-

Primary lymphedema, psoriasis, Vascular endothelial growth factor (VEGF), gene therapy

\begin{abstract}
Lymphedema is a chronic and debilitating pathology due to lymphatic stasis, responsible for an increase in the volume of the affected limb. Primary and secondary lymphedemas are distinguished. The former are due to a primitive anomaly of the lymphatic vessels (hereditary or not), the latter to their obstruction or destruction. Primary lymphedemas are classified as congenital, early and late by age of onset. Apart from functional and psychological repercussions, erysipelas remains the main complication, aggravating lymphatic circulatory deficit. The treatment is based on physical methods, resection surgery or reconstruction, and drugs (diuretic type, benzopyrones, coumarinic and flavonoids). These treatments are sometimes heavy or insufficient, and the therapeutic hope lies in gene therapy. We report here the case of psoriasis occurring in a patient presenting a primary lymphedema.
\end{abstract}

Copy Right, IJAR, 2018,. All rights reserved.

\section{Introduction:-}

Une femme de 45 ans suivie pour lymphoedéme (LO) primaire précoce; apparu vers l'âge de 16 ans; bilatéral des membres inferieurs (fig.1), sans autres malformations ni antécédents familiaux, consulte pour des lésions érythémato-squameuses prurigineuses apparues depuis 03 mois au niveau du cuir chevelu, du tronc et des mains, sans notion de prise médicamenteuse. À L'examen clinique la patiente était en surpoids avec un IMC à 26 , et l'examen cutané a objectivé des plaques érythémato-squameuses du dos, des mains (phalanges distales) et des pieds (fig.2, fig.1), ainsi qu'un intertrigo inter-fessier (fig.3), une omphalite (fig.4) et une onychodystrophie. Les examens biologiques ont révélé une hypercholestérolémie à $2,5 \mathrm{~g} / \mathrm{L}$ et une hyperglycémie non diabétique à $1,18 \mathrm{~g} / \mathrm{L}$. L'examen mycologique était négatif, et l'examen histo-pathologique était en faveur d'un psoriasis. La patiente a été mise sous régime alimentaire, Dermocorticoïde et émollient avec une bonne évolution clinique.

\section{Discussion:-}

Les LO des membres sont dus à une stase de la lymphe secondaire à un dysfonctionnement du système lymphatique. Ils sont classés schématiquement en primaires et secondaire à des lésions des voies lymphatiques. La prévalence de la population du LO est estimée entre 1,3 et 1,4 pour 1000 [1]. Le LO primaire est moins fréquent que le LO secondaire (filariose, traumatisme, thérapie anticancéreuse). Les LO primaires sont classés en congénitaux, précoces et tardifs en fonction de leur âge d'apparition (avant un an, entre un et 35 ans et après 35 ans, respectivement). Parmi ces LO primaires, on retrouve les formes familiales qui comprennent la maladie de Milroy (lymphœdème primaire congénital), la maladie de Meige ou encore le lymphœdème-distichiasis (précoces). Les LO primaires sont consécutifs à des anomalies génétiques dont l'étude a largement contribué à la compréhension de la 
lymphangiogenèse. Des études dans des familles ayant des formes héréditaires de LO ont identifié six gènes impliqués: FLT4 (codant pour VEGFR-3, impliqué dans la maladie de Milroy et situé en 5q34-35), FOXC2 (impliqué dans le syndrome distichiasis-lymphœdème et situé dans la région 16q24.1), SOX18 (20q13.33), HGF (7q21.11), MET (7q31.2) et CCBE1 (18q21.32) [2, 3, 4, 5, 6, 7, 8, 9]. Dans le syndrome de Meige le gène suspecté est GJC2 (Chromosome 1q41-q42). L'hétérogénéité génétique des LO primaires est une réalité, mais il est probable que ces gènes codent pour des protéines impliquées dans la cascade de signalisation du VEGFR-3 (Vascular endothelial growth factor receptor) ou des protéines de régulation de cette voie. Le VEGFR-3, ainsi que le ligand VEGF-C sont nécessaires à la mise en place du système lymphatique. Chez l'adulte, l'expression de VEGFR-3 est restreinte à l'endothélium lymphatique, aux endothéliums fenestrés et aux tissus cancéreux comme le sarcome de Kaposi et le cancer du sein $[\mathbf{1 0}, \mathbf{1 1}]$. In vitro, c'est un facteur essentiellement lymphangiogénique.

La pathogenèse du psoriasis quant à elle est un jeu complexe où sont impliqués les facteurs génétiques et environnementaux, les réponses immunitaires adaptées et innées et les principales cellules inflammatoires et épidermiques qui entraînent la maladie. La prolifération épidermique et capillaire semble être stimulée par la libération de cytokines à partir de lymphocytes.

Les dernières avancées génétiques clés comprennent la localisation chromosomique du locus majeur de susceptibilité au psoriasis, PSORS1 (psoriasis susceptibility 1), à 6p21-3. PSORS1 contribue jusqu'à 50\% du risque génétique $[12,13]$. Ont également été mis en évidence d'autres loci dont certains sont localisés sur les mêmes chromosomes impliqués dans les LO primaires: 1q21 (PSORS4), 1p (PSORS7), 16q12 (PSORAS1), 18p11, 20p.

Des altérations transcriptionelles ont été décrites pour des gènes codant pour des cytokines impliquées dans l'angiogenèse, notamment le VEGF, PDGF (platelet-derived growth factor) [14-15]. Ces dernières altérations sont responsables d'une croissance accrue des capillaires les plus superficiels chez les patients atteints d'un psoriasis. Les facteurs clés de stimulation de la lymphangiogénèse (VEGF-C et VEGF-D) sont produits par des macrophages transformés dans le derme (Schoppmann et al., 2002) et les kératinocytes dans l'épiderme (Huggenberger, 2010). Des études expérimentales ont révélé que la lymphangiogénèse peut également être induite par le VEGF-A, bien que les lymphatiques induits par le VEGF-A soient structurellement anormaux et fonctionnellement insuffisants (Nagy et al., 2002). Au contraire, la stimulation de la lymphangiogenèse par le VEGF-C inhibe de manière significative l'inflammation chronique de la peau (Huggenberger et al., 2010). On peut affirmer que les vaisseaux lymphatiques nouvellement formés dans l'inflammation chronique permettent une réduction de l'œdème tissulaire et augmentent les réponses immunitaires en favorisant le recrutement des macrophages et des cellules dendritiques (Baluk et al., 2005, Kataru et al., 2009). Il est supposé que l'inflammation chronique, l'angiogenèse, la lymphangiogenèse et l'hyperplasie épidermique dans les lésions psoriasiques s'influencent mutuellement et qu'elles fonctionnent de manière très régulée. Cette relation est appuyée par beaucoup d'études, mais les conséquences physiopathologiques détaillées et les mécanismes moléculaires sous-jacents à cette association ne sont pas entièrement compris et ils ont besoin d'une enquête plus approfondie. L'interaction entre ces processus pourrait être un avantage potentiel pour les approches thérapeutiques car l'inhibition de l'angiogenèse et paradoxalement l'activation de la lymphangiogénèse pourrait servir de stratégie nouvelle pour traiter l'inflammation chronique. À l'inverse, le ciblage de l'inflammation peut diminuer la production d'angiogenèse et de stimulants de la lymphangiogénèse. Des résultats prometteurs ont été obtenus à partir de modèles transgéniques de souris du psoriasis où le traitement anti-VEGF systémique a considérablement réduit l'inflammation de la peau (Jackson et al., 1997; Costa et al., 2007; Schonthaler et al., 2009). Chez la souris mutante Chy, développant un LO des pattes et une ascite chyleuse comme chez l'homme atteint de LO héréditaire congénital, l'administration de VEGF-C induit la croissance des vaisseaux lymphatiques au niveau cutané et ainsi la disparition du LO des pattes [16].

La limite à ces thérapeutiques reste l'implication du système lymphatique et du VEGF-C/VEGFR-3 dans la cancérogenèse et l'extension métastatique chez l'homme [11], mais ces observations ouvrent des perspectives thérapeutiques génétiques et biologiques innovantes tant pour le LO qu'en ce qui concerne le psoriasis et l'extension métastatique en cancérologie. 


\section{Iconographie:-}

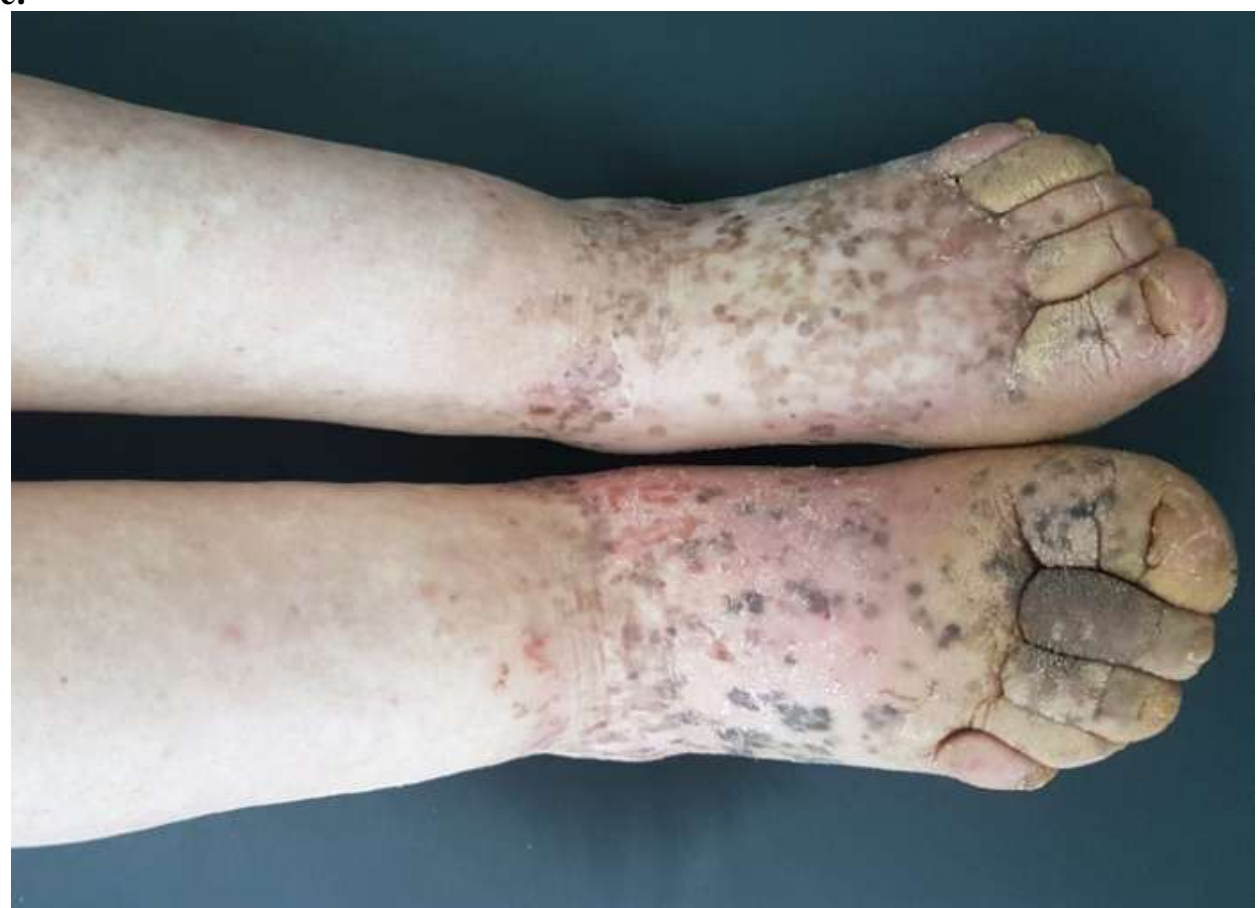

Fig.1:-lymphoedeme des jambes avec plaques érythémato-squameuses des pieds

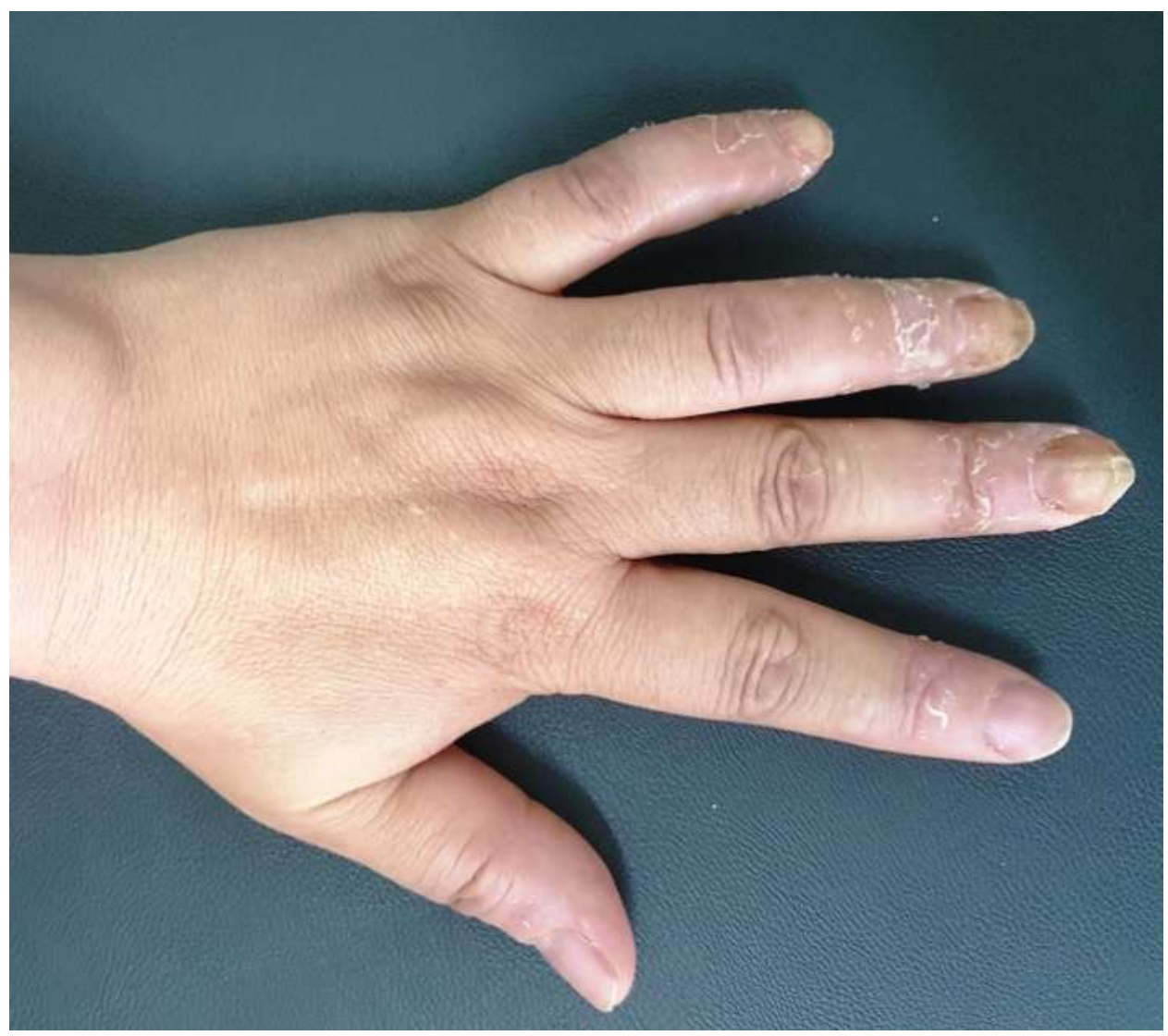

Fig.2:-lésions érythémato-squameuses des phalanges distales avec onychodystrophie. 


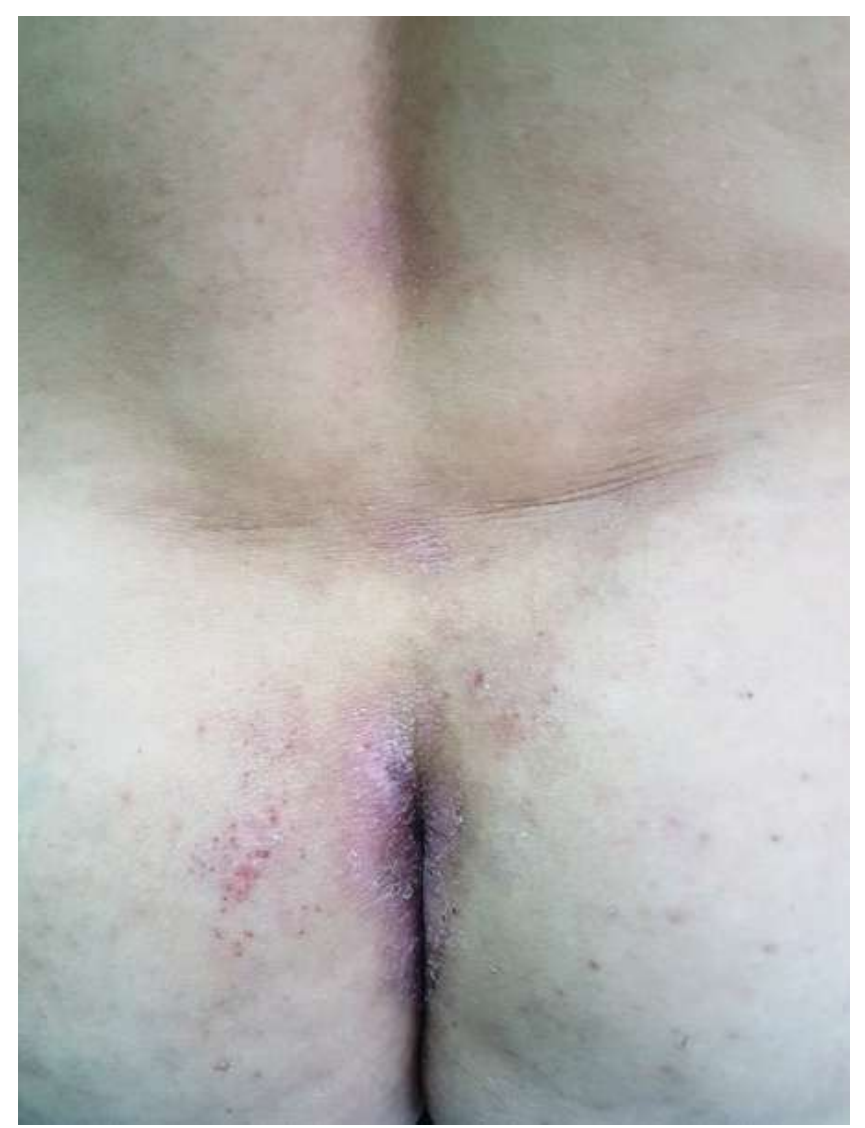

Fig.3:-plaques érythémato-squameuses du dos avec un intertrigo inter-fessier

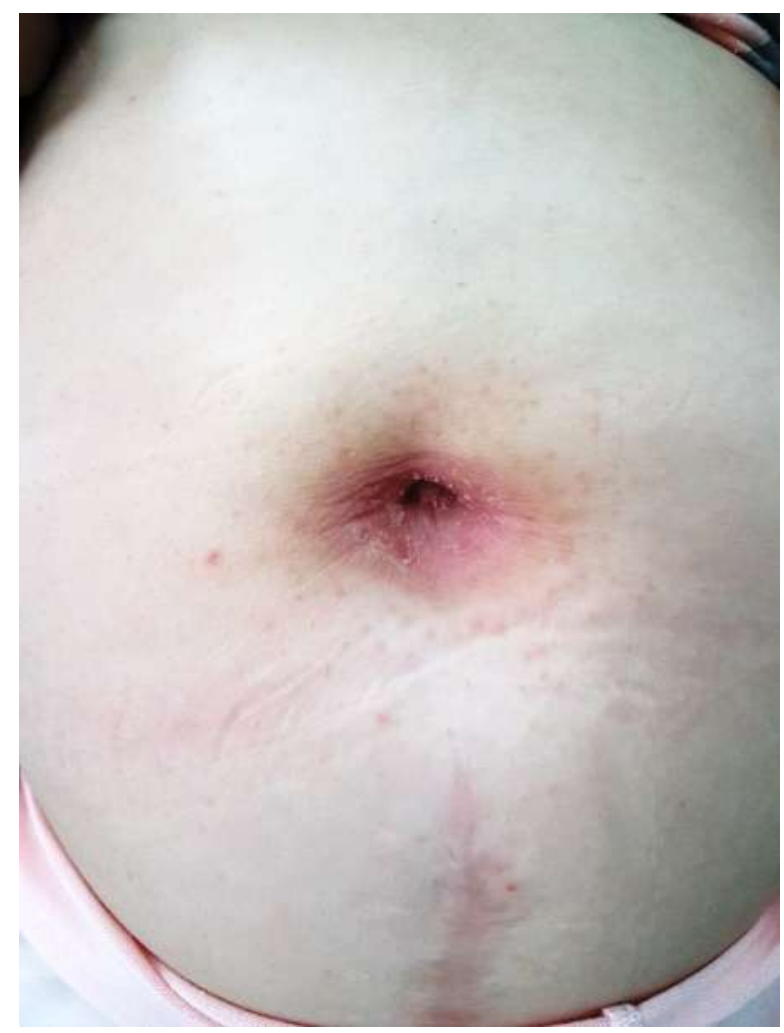

Fig.4:-omphalite psoriasique. 


\section{References:-}

1. Rockson, S.G., and Rivera, K.K. (2008). Estimating the population burden of lymphedema. Ann. N Y Acad. Sci. 1131, 147-154

2. Ferrell, R.E., Levinson, K.L., Esman, J.H., Kimak, M.A., Lawrence, E.C., Barmada, M.M., and Finegold, D.N. (1998). Hereditary lymphedema: evidence for linkage and genetic heterogeneity. Hum. Mol. Genet. 7, 20732078.

3. Karkkainen, M.J., Ferrell, R.E., Lawrence, E.C., Kimak, M.A., Levinson, K.L., McTigue, M.A., Alitalo, K., and Finegold, D.N. (2000). Missense mutations interfere with VEGFR-3 signalling in primary lymphoedema. Nat. Genet. 25, 153-159.

4. Fang, J., Dagenais, S.L., Erickson, R.P., Arlt, M.F., Glynn, M.W., Gorski, J.L., Seaver, L.H., and Glover, T.W. (2000). Mutations in FOXC2 (MFH-1), a forkhead family transcription factor, are responsible for the hereditary lymphedema-distichiasis syndrome. Am. J. Hum. Genet. 67, 1382-1388.

5. Finegold, D.N., Kimak, M.A., Lawrence, E.C., Levinson, K.L., Cherniske, E.M., Pober, B.R., Dunlap, J.W., and Ferrell, R.E. (2001). Truncating mutations in FOXC2 cause multiple lymphedema syndromes. Hum. Mol. Genet. 10, 11851189

6. Irrthum, A., Devriendt, K., Chitayat, D., Matthijs, G., Glade, C., Steijlen, P.M., Fryns, J.P., Van Steensel, M.A., and Vikkula, M. (2003). Mutations in the transcription factor gene SOX18 underlie recessive and dominant forms of hypotrichosis-lymphedema-telangiectasia. Am. J. Hum. Genet. 72, 1470-1478.

7. Finegold, D.N., Schacht, V., Kimak, M.A., Lawrence, E.C., Foeldi, E., Karlsson, J.M., Baty, C.J., and Ferrell, R.E. (2008). HGF and MET mutations in primary and secondary lymphedema. Lymphat. Res. Biol. 6, 65-68

8. Alders, M., Hogan, B.M., Gjini, E., Salehi, F., Al-Gazali, L., Hennekam, E.A., Holmberg, E.E., Mannens, M.M., Mulder, M.F., Offerhaus, G.J., et al. (2009). Mutations in CCBE1 cause generalized lymph vessel dysplasia in humans. Nat. Genet. 41, 1272-1274.

9. Connell, F., Kalidas, K., Ostergaard, P., Brice, G., Homfray, T., Roberts, L., Bunyan, D.J., Mitton, S., Mansour, S., Mortimer, P., and Jeffery, S.; Lymphoedema Consortium. (2010). Linkage and sequence analysis indicate that CCBE1 is mutated in recessively inherited generalised lymphatic dysplasia. Hum. Genet. 127, 231-241

10. Partanen TA, Arola J, Saaristo A, Jussila L, Ora A, Miettinen M, et al. VEGF-C and VEGF-D expression in neuroendocrine cells and their receptor, VEGFR-3 in fenestrated blood vessels in human tissues. FASEB J 2000 ; 14 : 2087-96. 2000 ; 14 : 2087-96.

11. Valtola R, Salven P, Heikkila P, Taipale J, Joensuu H, Rehn M, et al. VEGFR-3 and its ligand VEGF-C are associated with angiogenesis in breast cancer. Am J Pathol 1999; 154 : 1381 - 90.

12. F.Capon M.Munro J.Barker R.Trembath: Searching for the major histocompatibility complex psoriasis susceptibility gene. J Invest Dermatol1182002745751.

13. Trembath RC, Clough RL, Rosbotham JL, Jones AB, Camp RD, Frodsham A, et al. Identification of a major susceptibility locus on chromosome $6 \mathrm{p}$ and evidence for further disease loci revealed by a two stage genomewide search in psoriasis. Hum Mol Genet 1997;6:813-20.

14. Bowcock AM, Krueger JG. Getting under the skin: the immunogenetics of psoriasis. Nat Rev Immunol 2005;5:699-711

15. Viac J, Palacio S, Schmitt D, Claudy A. Expression of vascular endothelial growth factor in normal epidermis, epithelial tumors and cultured keratinocytes. Arch Dermatol Res 1997;289:158-63

16. Karkkainen MJ, Saaristo A, Jussila L, Karila KA, Lawrence EC, Pajusola K, et al. A model for gene therapy of human hereditary lymphedema. Proc NatlAcad Sci USA2001 ; 98 : 12677- 82. 